\title{
On the Unification of Process Semantics: Observational Semantics ${ }^{\star}$
}

\author{
David de Frutos Escrig, Carlos Gregorio Rodríguez, and Miguel Palomino \\ Departamento de Sistemas Informáticos y Computación, UCM
}

\begin{abstract}
The complexity of parallel systems has produced a large collection of semantics for processes. Van Glabbeek's linear time-branching time spectrum provides a classification of most of these semantics; however, no suitable unified definitions were available. We have discovered how to unify them, both in an observational framework and in an equational framework. In this first part of our study we present the observational semantics, that stresses the differences between the simulation (branching) semantics and the extentional (linear) semantics. As a result we rediscover the classification in van Glabbeek's spectrum and shed light on it, obtaining a framework where we can consider all the semantics in the spectrum at the same time. Also, we have discovered some "lost links" that correspond to semantics, possibly not too interesting (at the moment), that provide a clearer picture of the spectrum.
\end{abstract}

\section{Introduction}

The complexity of parallel systems has given rise to a large collection of semantics for processes, whose diversity is mainly due to the way in which non-determinism is treated. Most of these semantics have been compiled into van Glabbeek's lineartime branching-time (ltbt) spectrum [6], where they are first presented along the lines of their original definitions and are then characterized in three frameworks: observational/testing, logical, and by means of finite axiomatizations whenever possible. However, even when presented in a common framework these definitions are hard to compare with each other because the testing scenarios vary widely or the different sets of axioms appear completely unrelated.

After several years studying process semantics searching for homogeneous presentations we have discovered a way to unify them, both within an observational and an equational framework. Here we focus on the observational semantics, according to which we have classified the process semantics in four classes:

- bisimulation semantics, which is the only one that cannot be defined by means of a non-trivial preorder;

- the simulation semantics ( simulation, complete simulation, ready simulation, nested simulation, ... ) characterized by means of branching observations, that is, labeled trees;

\footnotetext{
* Research supported by the Spanish projects DESAFIOS TIN2006-15660-C02-02, WEST TIN2006-15578-C02, and the Comunidad de Madrid project PROMESAS-CAM S-0505/ TIC/0407.
} 
- the linear semantics (traces, failures, readiness, ... ), characterized by linear observations, a degenerated case of branching observations;

- the deterministic branching semantics corresponding to an intermediate class between branching and linear, where observations are deterministic trees. Possible worlds semantics is the only semantics in the spectrum in this class.

Besides their linear or branching nature, semantics are characterized by a local observation function that generates the local observations of the states. For the linear case there is also the possibility of observing this local information in a partial way and this is how for each local observer, in principle, up to four different semantics can be obtained. In particular, this gives rise to the classic diamond below the ready simulation semantics formed by the failures, failure-traces, readiness, and ready-traces semantics.

Our uniform presentation of the process semantics will clarify the relations and hierarchies among them; moreover, it will make generic proofs of their properties possible. In particular, we have obtained a uniform presentation of their axiomatizations that we study in detail in a second part to this paper [3]. The proofs of all results presented in this paper, together with some additional material, can be found in the extended version available at http://maude.sip.ucm.es/ miguelpt/

We are aware of the fact that there exists a very extensive literature on the field of semantics for concurrency and, in particular, on the ltbt spectrum but, due to lack of space we can only cite here some representative papers. Without any doubt [6], as commented above, is the key work on the subject, and in fact our guiding motivation is to complete it, providing a more uniform description of the ltbt spectrum; that work contains references to all the original presentations of the semantics in the spectrum. There have also been several efforts aimed at developing generic frameworks in which all those semantics could be uniformly presented. Since we are specially interested in the relation between the simulation (branching time) semantics and those based on decorated traces (linear time), we recall here the recent work by Jacobs [5] where he develops trace semantics in a coalgebraic framework, and that by Boreale and Gaducci [1], giving a coinductive presentation of failures semantics.

\section{Preliminaries}

Although the main results in this paper are valid for infinite processes, to simplify the presentation we will mainly consider finite processes generated by the basic process algebra BCCSP.

Definition 1. Given a set of actions Act, the set BCCSP (Act) of processes is defined by the following BNF-grammar:

$$
p::=\mathbf{0}|a p| p+q
$$

where $a \in A c t ; 0$ represents the process that performs no action; for every action in Act, there is a prefix operator; and + is a choice operator.

The operational semantics for BCCSP terms is defined by

$$
\text { ap } \stackrel{a}{\longrightarrow} p \quad \frac{p \stackrel{a}{\longrightarrow} p^{\prime}}{p+q \stackrel{a}{\longrightarrow} p^{\prime}} \quad \frac{q \stackrel{a}{\longrightarrow} q^{\prime}}{p+q \stackrel{a}{\longrightarrow} q^{\prime}}
$$

As usual, we write $p \stackrel{a}{\longrightarrow}$ if there exists a process $q$ such that $p \stackrel{a}{\longrightarrow} q$. 
Many different semantics for these non-deterministic processes have been defined in the literature. The most important and popular semantics appear in van Glabbeek's spectrum [6]. One indirect way to capture any semantics is by means of the equivalence relation induced by it: given a formal semantics $\left[[\cdot]_{X}\right.$, we say that processes $p$ and $q$ are equivalent iff they have the same semantics, that is, $p \equiv_{X} q \Leftrightarrow\left[[p]_{X}=\left[[q]_{X}\right.\right.$. Also, these semantics can be defined by means of adequate observational scenarios, or by logical characterisations that introduce natural preorders $\Xi_{X}$ whose kernels are the semantic equivalences. For instance, we will write $\bigsqcup_{R S}$ for ready simulation, $\bigsqcup_{F}$ for failures, and so on. We refer to [6] for the original definition and usual notation for all the semantics in the ltbt spectrum that will be discussed throughout the paper.

Bisimilarity (denoted with $\equiv$ ), the strongest of the semantics in the spectrum, can be axiomatized by means of the four simple axioms

$$
\begin{array}{ll}
\left(B_{1}\right) x+y \simeq y+x & \left(B_{3}\right) x+x \simeq x \\
\left(B_{2}\right)(x+y)+z \simeq x+(y+z) & \left(B_{4}\right) x+\mathbf{0} \simeq x
\end{array}
$$

These axioms state that the choice operator is commutative, associative and idempotent, having the empty process as identity element. These axioms also justify the use of the notation $\sum_{a} \sum_{i} a p_{a}^{i}$ for processes, where the commutativity and associativity of the choice operator is used to group together the summands whose initial action is $a$.

The initial offer of a process is the set $I(p)=\{a \mid a \in$ Act and $p \stackrel{a}{\longrightarrow}\}$. This is a simple, but quite important observation function that plays a central role in the definition of the most popular semantics in the linear time-branching time spectrum. We will also denote by $I$ the relation expressing the fact that two processes have the same initial offer: $p I q \Leftrightarrow I(p)=I(q)$.

Some of the semantics in the spectrum are constrained simulation semantics that can be defined in a parameterized way.

Definition 2. Given a relation $N$ over BCCSP processes, an $N$-constrained simulation is a relation $S_{N}$ such that $p S_{N} q$ implies:

- For every a, if $p \stackrel{a}{\longrightarrow} p^{\prime}$ there exists $q^{\prime}, q \stackrel{a}{\longrightarrow} q^{\prime}$ and $p^{\prime} S_{N} q^{\prime}$, and

- $p N q$.

We say that process $p$ is $N$-simulated by process $q$, or that $q N$-simulates $p$, written $p \sqsubseteq_{N S} q$, whenever there exists an $N$-constrained simulation $S_{N}$ such that $p S_{N} q$.

We have already studied the constrained simulation semantics in detail in [2], stressing their general properties. In particular, the following constraints are considered: the universal relation $U$ relating all processes, which gives rise to the simulation semantics; the relation $C$, which holds for processes $p$ and $q$ when both, or none, are isomorphic to $\mathbf{0}$, and that gives rise to the complete simulation semantics; $I$, which corresponds to ready simulation; $T$, that relates processes with the same traces and corresponds to trace simulation; $S$, the inverse of the simulation relation, whose associated constrained simulation is the 2-nested simulation.

Besides the semantics in the spectrum, we are interested in a general study covering any reasonable semantics coarser than bisimilarity. Since we will use preorders to characterise these semantics we introduce the following definitions that state the desired properties of those reasonable preorders. 
Definition 3. A preorder relation $\sqsubseteq$ over processes is a behavior preorder if

- it is weaker than bisimilarity, i.e. $p \equiv_{B} q \Rightarrow p \sqsubseteq q$, and

- it is a precongruence with respect to the prefix and choice operators, i.e. if $p \sqsubseteq q$ then ap $\sqsubseteq a q$ and $p+r \sqsubseteq q+r$.

If $\sqsubseteq$ is actually an equivalence, it is called behavior equivalence.

\section{Branching General Observations}

In order to characterize the simulation semantics in an extensional way we need local and branching general observations.

Definition 4. The sets $L_{N}$ of local observations corresponding to each of the $N$-constrained simulations in the spectrum, and $L_{N}(p)$ of observations associated to a process $p$, are defined as follows:

- Plain simulation: $L_{U}=\{\cdot\}, L_{U}(p)=\cdot$.

- Ready simulation: $L_{I}=\mathscr{P}($ Act $), L_{I}(p)=I(p)$.

- Complete simulation: $L_{C}=$ Bool, $L_{C}(p)$ is true if $p \equiv \mathbf{0}$ and false otherwise.

- Trace simulation: $L_{T}=\mathscr{P}\left(A c t^{*}\right), L_{T}(p)$ is $T(p)$, the set of traces of $p$.

- 2-nested simulation: $L_{S}=\left\{[[p]]_{S} \mid p \in B C C S P\right\}, L_{S}(p)=[[p]]_{S}$.

- n-nested simulation: $L_{S}=\left\{\left[[p]_{(n-1) S} \mid p \in B C C S P\right\}, L_{S}(p)=\left[[p]_{(n-1) S}\right.\right.$, where $[[p]]_{k S}$ denotes the $k$-nested simulation equivalence class of $p$.

Definition 5. 1. A branching general observation (bgo for short) of a process is a finite, non-empty tree whose arcs are labeled with actions in Act and whose nodes are labeled with local observations from $L_{N}$, for $N$ a constraint; the corresponding set $B G O_{N}$ is recursively defined as:

- $\langle l, \emptyset\rangle \in B G O_{N}$ for $l \in L_{N}$.

- $\left\langle l,\left\{\left(a_{i}\right.\right.\right.$, bgo $\left.\left.\left._{i}\right) \mid i \in 1 . . n\right\}\right\rangle \in B G O_{N}$ for every $n \in \mathbb{N}, a_{i} \in$ Act and $b g o_{i} \in B G O_{N}$.

2. The set $B G O_{N}(p)$ of branching general observations of $p$ corresponding to the constraint $N$ is

$$
B G O_{N}(p)=\left\{\left\langle L_{N}(p), S\right\rangle \mid S \subseteq\left\{(a, b g o) \mid b g o \in B G O_{N}\left(p^{\prime}\right), p \stackrel{a}{\longrightarrow} p^{\prime}\right\}\right\} .
$$

3. We write $p \leq_{N}^{b} q$ if $B G O_{N}(p) \subseteq B G O_{N}(q)$.

In Figure 1 some simple examples of bgo's for $N=I$ are shown. We have represented bgo $_{1}$ as $\langle\{a\},\{(a,\langle\{b\},\{(b,\langle\{c\}, \emptyset\rangle)\}\rangle),(a,\langle\{b\},\{(b,\langle\{d\}, \emptyset\rangle)\}\rangle)\}\rangle$ and bgo $_{2}$ as $\langle\{a\},\{(a,\langle\{b\},\{(b,\langle\{c\}, \emptyset\rangle),(b,\langle\{d\}, \emptyset\rangle)\}\rangle)\}\rangle$. We use braces for the set of children of a node, parentheses to represent a branch of the tree as a pair (initial arc, subtree below), and angle brackets to represent each tree as a pair 〈root, children〉.

Example 1. For $N=I$, if $x=b(c+d)$ and $y=b c+b d$, then for $p=a(x+y)$ we have $b g o_{k} \in B G O_{I}(p)$ for $k \in\{1,2,3\}$, where the bgo's are depicted in Figure 2 It is easy to check that all of them are also branching observations of $q=a(x+y)+a x$. As a matter of fact, we have $B G O_{I}(p)=B G O_{I}(q)$. Note that in order to have $b g o_{3} \in B G O_{I}(p)$ we 


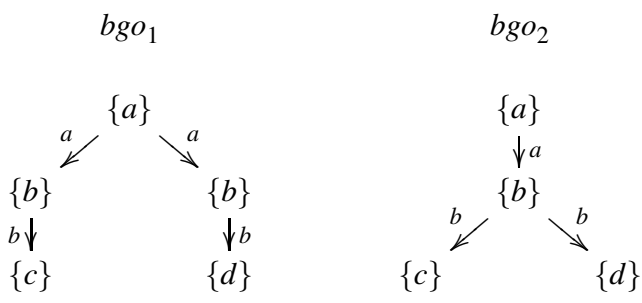

Fig. 1. Two branching observations

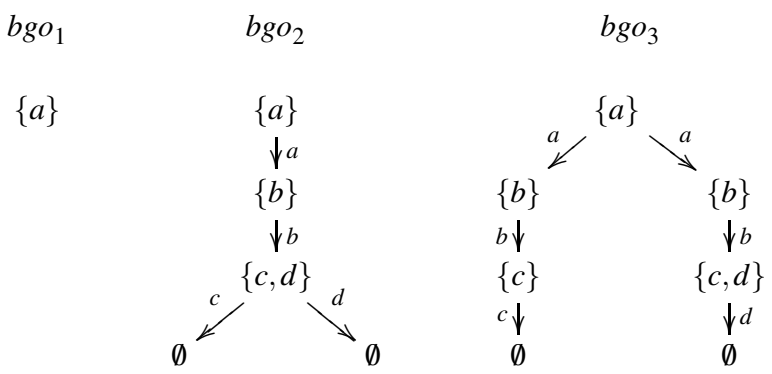

Fig. 2. Three branching observations

need to consider two different observations of the process $x+y$, which is the only $p^{\prime}$ such that $a(x+y) \stackrel{a}{\longrightarrow} p^{\prime}$.

By contrast, for $p=a(b c+b d)$ and $q=a b c+a b d, B G O_{I}(q) \nsubseteq B G O_{I}(p)$, since for the branching observation $b g o_{1}$ in Figure 1 we have $b g o_{1} \in B G O_{I}(q)$ and $b g o_{1} \notin$ $B G O_{I}(p)$. And also, we have $B G O_{I}(p) \nsubseteq B G O_{I}(q)$ since for $b g o_{2}$ as in Figure 1 we have $b g o_{2} \in B G O_{I}(p)$ but $b g o_{2} \notin B G O_{I}(q)$. The key idea is that, following our definition of bgo, we can include in a single bgo two separated computations but we cannot mix two different ones, even if the labels both in their initial transitions and in the local observations of the reached nodes were the same.

Let us also comment on the fact that in all five cases that we have considered in Definition 4 the local observation functions $L_{N}$ define a representation of the equivalence relation $N$ used to define the constrained simulation relations. This means that we have $L_{N}(p)=L_{N}(q) \Longleftrightarrow p N q$.

The fact that the observational semantics $B G O_{N}(p)$ can be defined in a compositional way will simplify the proofs of many of their properties.

Theorem 1. Let $L$ be a function used as local observation function such that there exist semantical functions ${ }^{L}{ }^{L}: L_{N} \times L_{N} \rightarrow L_{N}$ and $a^{L}: L_{N} \rightarrow L_{N}$ satisfying $L(a p)=a^{L} L(p)$ and $L(p+q)=L(p)+{ }^{L} L(q)$. Then:

$$
\begin{array}{r}
-B G O_{N}(a p)=\left\{\left\langle a^{L} L(p),\{(a, b g o) \mid \text { bgo } \in B\}\right\rangle \mid B \subseteq B G O_{N}(p)\right\} . \\
\text { - } B G O_{N}(p+q)=\left\{\left\langle L(p)+{ }^{L} L(q), S_{1} \cup S_{2}\right\rangle \mid\left\langle L(p), S_{1}\right\rangle \in B G O_{N}(p),\right. \\
\left.\left\langle L(p), S_{2}\right\rangle \in B G O_{N}(q)\right\} .
\end{array}
$$

In particular, $B G O_{N}(p)$ is compositional for our fundamental constraints. 
Proposition 1. For all $N \in\{U, I, C, T, S\}, L_{N}$ can be defined in a compositional way over the terms in BCCSP.

Now we show that bgo's characterize $N$-simulation semantics in all cases.

Theorem 2. For all $N \in\{U, I, C, T, S\}$ and any two processes $p$ and $q, p \sqsubseteq_{N S} q$ iff $p \leq_{N}^{b} q$.

In principle, any behavior preorder could be used as such a constraint $N$. For instance, the predicate $I_{\subseteq}$ defined by $I_{\subseteq}(p, q)$ iff $I(q) \subseteq I(p)$. But from $I(q) \subseteq I(p)$ we cannot conclude that $L_{N}(p)=L_{N}(q)$ and, hence, either a more complicated characterization of $\sqsubseteq_{N S}$ in terms of bgo's or an additional argument to show that $p \sqsubseteq_{I_{\subseteq}} q$ implies $I(p) \subseteq I(q)$ would be needed: this is why it is always advisable to consider equivalence behaviors as constraints.

Corollary 1. For any constraint $N$ that is a behavior equivalence, whenever we have as local observation function $L_{N}$ the quotient function $L_{N}(p)=\left[[p]_{N}\right.$ or any concrete representation of it satisfying $L_{N}(p)=L_{N}(q) \Longleftrightarrow N(p, q)$, then $p \sqsubseteq_{N S} q$ iff $B G O_{N}(p) \subseteq B G O_{N}(q)$.

The results above bring forward the fact that despite the similarity between the bgo's of a process and its computation tree, the possibility of mixing several computations in a single branching observation makes it possible to identify non-bisimilar processes by their sets of branching observations.

\section{Linear Observations and Linear Time Semantics}

We introduce the linear observations of a process as a particular (degenerate) case of branching observations: those with a linear structure.

Definition 6. 1. The set $L G O_{N}$ of linear general observations (lgo for short) for a local observer $L_{N}$ is the subset of $B G O_{N}$ defined as:

- $\langle l, \emptyset\rangle \in L G O_{N}$ for each $l \in L_{N}$.

- $\langle l,\{(a$, lgo $)\}\rangle$, whenever $a \in A$ and lgo $\in L G O_{N}$.

2. The set of linear general observations of a process $p$ with respect to the local observer $L_{N}$ is $L G O_{N}(p)=B G O_{N}(p) \cap L G O_{N}$.

Since lgo's are linear they can be presented as traces, avoiding the sets of descendants in the general bgo's. Therefore, we will consider them as elements of the set $L_{N} \times($ Act $\times$ $\left.L_{N}\right)^{*}$.

It is also clear that the set of linear observations can be defined recursively without resorting to branching observations.

Proposition 2. The set $\mathrm{LGO}_{N}(p)$ of linear general observations of a process $p$ is recursively defined by

$$
L G O_{N}(p)::=\left\{\left\langle L_{N}(p)\right\rangle\right\} \cup\left\{\left\langle L_{N}(p), a\right\rangle \circ \lg o \mid p \stackrel{a}{\longrightarrow} p^{\prime}, \lg o \in L G O_{N}\left(p^{\prime}\right)\right\}
$$

We can also compute $L G O_{N}(p)$ in a compositional way. 
Proposition 3. Let $L$ be a local observation function such that there exist semantical functions $+^{L}: L_{N} \times L_{N} \rightarrow L_{N}$ and $a^{L}: L_{N} \rightarrow L_{N}$ satisfying $L(a p)=a^{L} L(p)$ and $L(p+$ $q)=L(p)+{ }^{L} L(q)$. Then:

$$
\begin{gathered}
-L G O_{N}(a p)=\left\{\left\langle a^{L} L(p)\right\rangle\right\} \cup\left\{\left\langle a^{L} L(p), a\right\rangle \circ L G O_{N}(p)\right\} . \\
-L G O_{N}(p+q)=\left\{\left\langle L(p)+{ }^{L} L(q)\right\rangle \circ t \mid\langle L(p)\rangle \circ t \in L G O_{N}(p)\right. \text { or } \\
\left.\langle L(p)\rangle \circ t \in L G O_{N}(q)\right\} .
\end{gathered}
$$

Obviously, $L G O_{U}$ is isomorphic to $A c t^{*}$ for $N=U$ and, thus, $L G O_{U}(p)=\operatorname{Traces}(p)$. By contrast, for $N=I, L G O_{I}(p)$ is the set of ready traces of $p, \operatorname{ReadyTraces}(p)$.

Set inclusion of the sets of linear observations with respect to a local observer $L_{N}$ gives us the preorder defining the corresponding semantics.

Definition 7. A process $p$ is less than or equal to $q$ with respect to the linear observations generated by $L_{N}$, denoted $p \leq_{N}^{l} q$ if $L G O_{N}(p) \subseteq L G O_{N}(q)$. We will denote the corresponding equivalence by $=_{N}^{l}$.

Proposition 4. (1) $\leq_{U}^{l}=\sqsubseteq_{T} ;(2) \leq_{I}^{l}=\sqsubseteq_{R T} ;(3) \leq_{C}^{l}=\sqsubseteq_{C T}$.

Proposition 5. For all $N \in\{U, C, I, T, S\}$, if $p \sqsubseteq_{N S} q$ then $p \leq_{N}^{l} q$, but the converse may not hold.

Therefore, by means of linear observations and set inclusion, we can characterize the orders that define some of the semantics in the spectrum which are not simulation semantics. However, there are still some other semantics for which a different way of treating the linear observations is needed.

Definition 8. For $\mathscr{T}, \mathscr{T}^{\prime} \subseteq L G O_{I}$ we define the orders $\leq_{I}^{l \supseteq}, \leq_{I}^{l f}$, and $\leq_{I}^{l f} \supseteq$ by:

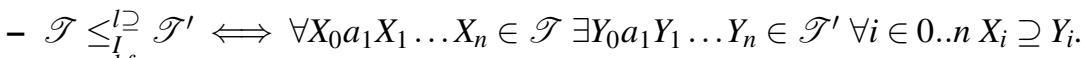

$$
\begin{aligned}
& \text { - } \mathscr{T} \leq_{I}^{l f} \mathscr{T}^{\prime} \Longleftrightarrow \forall X_{0} a_{1} X_{1} \ldots X_{n} \in \mathscr{T} \exists Y_{0} a_{1} Y_{1} \ldots Y_{n} \in \mathscr{T}^{\prime} X_{n}=Y_{n} \text {. }
\end{aligned}
$$

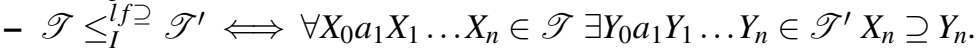

Then, we write $p \leq_{I}^{l X} q$ if $L G O_{I}(p) \leq_{I}^{l X} L G O_{I}(q)$.

Since the definition of $\leq_{I}^{l f}$ ignores all the intermediate ready sets $X_{i}$ with $i<n$ and requires the final ready sets to coincide, it defines the readiness preorder. Let us now prove that the two semantics based on failures are also characterized by our preorders $\leq_{I}^{l f} \supseteq$ and $\leq_{I}^{l \supseteq}$.

Proposition 6. The preorder $\leq_{I}^{l f} \supseteq$ generates the failures preorder and $\leq_{I}^{l \supseteq}$ generates the failures trace preorder.

As a matter of fact, the characterization of failures by means of the reverse inclusion of offerings is not a great discovery at all, and the same idea can be found in the definition of acceptance trees [4]. However, it is by means of our sets of linear observations that a quite nice characterization is obtained so that we can forget about the notion of failures and consider instead reverse inclusion of offerings. But the most important property of our characterizations in terms of different orders on the set $L G O_{I}$ is that they can be generalized to other local observation functions. 
Definition 9. For $\mathscr{T}, \mathscr{T}^{\prime} \subseteq L G O_{N}$ we define the orders $\leq_{N}^{l \supseteq}, \leq_{N}^{l f}$, and $\leq_{N}^{l f} \supseteq$ by:

$$
\begin{aligned}
& \text { - } \mathscr{T} \leq_{N}^{l \supseteq} \mathscr{T}^{\prime} \Longleftrightarrow \forall X_{0} a_{1} X_{1} \ldots X_{n} \in \mathscr{T} \exists Y_{0} a_{1} Y_{1} \ldots Y_{n} \in \mathscr{T}^{\prime} \forall i \in 0 . . n X_{i} \supseteq Y_{i} . \\
& \text { - } \mathscr{T} \leq_{N}^{l f} \mathscr{T}^{\prime} \Longleftrightarrow \forall X_{0} a_{1} X_{1} \ldots X_{n} \in \mathscr{T} \exists Y_{0} a_{1} Y_{1} \ldots Y_{n} \in \mathscr{T}^{\prime} X_{n}=Y_{n} . \\
& \text { - } \mathscr{T} \leq_{N}^{l f} \supseteq \mathscr{T}^{\prime} \Longleftrightarrow \forall X_{0} a_{1} X_{1} \ldots X_{n} \in \mathscr{T} \exists Y_{0} a_{1} Y_{1} \ldots Y_{n} \in \mathscr{T}^{\prime} X_{n} \supseteq Y_{n} \text {. }
\end{aligned}
$$

Then, we write $p \leq_{N}^{l X}$ q if $L G O_{N}(p) \leq_{N}^{l X} L G O_{N}(q)$.

By abuse of notation, we have used the superset inclusion symbol $\supseteq$ in the definitions above for all $N$. That is the right interpretation for the cases $N=I, T$; for $N=U, C$ the superset inclusions degenerate to equalities. For $N=S$, it should be interpreted as $[[p]]_{S} \geq_{S}\left[[q]_{S}\right.$. Then, it is easy to see that we could have used such an inequality $[[p]]_{N} \geq_{N}\left[[q]_{N}\right.$ in all cases.

For an observational semantics one expects that the order between processes is governed by set inclusion as is the case, for instance, for the classic definition of failures semantics. Fortunately, it is easy to obtain such a characterization for the three semantics considered above by means of suitable closure operators.

Definition 10. For $\mathscr{T} \subseteq L G O_{N}$, the following three closures are defined:

$$
\begin{aligned}
& \text { - } \overline{\mathscr{T}}^{\supseteq}=\left\{X_{0} a_{1} X_{1} \ldots a_{n} X_{n} \mid \exists Y_{0} a_{1} Y_{1} \ldots a_{n} Y_{n} \in \mathscr{T} \forall i \in 0 . . n X_{i} \supseteq Y_{i}\right\} . \\
& \text { - } \overline{\mathscr{T}}^{f}=\left\{X_{0} a_{1} X_{1} \ldots a_{n} X_{n} \mid \exists Y_{0} a_{1} Y_{1} \ldots a_{n} X_{n} \in \mathscr{T}\right\} . \\
& \text { - } \overline{\mathscr{T}}^{f \supseteq}=\left\{X_{0} a_{1} X_{1} \ldots a_{n} X_{n} \mid \exists Y_{0} a_{1} Y_{1} \ldots a_{n} Y_{n} \in \mathscr{T} X_{n} \supseteq Y_{n}\right\} .
\end{aligned}
$$

Then, if $X \in\{\supseteq, f, f \supseteq\}$, for $p \in B C C S P$ and $N$ a constraint, we define $\operatorname{LGO}_{N}^{X}(p)=$ $\overline{L G O_{N}(p)}{ }^{X}$.

Proposition 7. All the operations in Definition 10 are indeed closures: if $X \in\{\supseteq, f, f \supseteq\}$ and $\mathscr{T}, \mathscr{T}^{\prime} \subseteq L G O_{N}$, then $\mathscr{T} \subseteq \overline{\mathscr{T}}^{X}$ and ${\overline{\mathscr{T}^{X}}}^{X}=\overline{\mathscr{T}}^{X}$; also, if $\mathscr{T} \subseteq \mathscr{T}^{\prime}$ then $\overline{\mathscr{T}}^{X} \subseteq$ $\overline{\mathscr{T}}^{\mathrm{X}}$.

Proposition 8. For all $X \in\{\supseteq, f, f \supseteq\}, \mathscr{T} \leq_{N}^{l X} \mathscr{T}^{\prime}$ iff $\overline{\mathscr{T}}^{X} \subseteq \overline{\mathscr{T}}^{X}$.

Let us see which of the semantics in the spectrum are characterized by the orders $\leq_{N}^{l X}$ above.

Proposition 9. For $N=U$ we have $\leq_{U}^{l}=\leq_{U}^{l \supset}=\leq_{U}^{l f}=\leq_{U}^{l f \supseteq}=\sqsubseteq_{T}$. As a consequence, the only semantics coarser than plain simulation that can be characterized by means of linear observations using $L_{U}$ is the trace semantics.

Proposition 10. For $N=C$ we have $\leq_{C}^{l}=\leq_{C}^{l \supseteq}=\leq_{C}^{l f}=\leq_{C}^{l f \supseteq}=\sqsubseteq_{C T}$. As a consequence, the only semantics coarser than complete simulation that can be characterized by means of linear observations using $L_{C}$ is the complete trace semantics.

Proposition 11. For $N=I, \leq_{I}^{l f} \supseteq$ characterizes the failures semantics, $\leq_{I}^{l f}$ the readiness semantics, $\leq_{I}^{l}$ the failure traces semantics, and $\leq_{I}^{l}$ the ready trace semantics. Therefore, the possible worlds semantics is the only semantics in the ltbt spectrum coarser than ready simulation that cannot be characterized using lgo $o_{I}$ 's. 
As we will see in Section 5, the possible world semantics is the only deterministic branching semantics in the spectrum and will require the use of the deterministic branching observations introduced there to be characterized in an observational way.

Proposition 12. $1 . \leq_{T}^{l f}$ is the possible futures preorder;

2 . $\leq_{T}^{l f} \supseteq$ is the impossible futures preorder.

As a matter of fact, the possible futures semantics is just below the 2-nested simulation semantics in the spectrum, only because the trace simulation semantics is missing there. The impossible futures semantics has been introduced quite recently [7] and is not yet well-known. And it is at this point where we have discovered our first two "lost creatures", defined as follows.

Definition 11. The possible futures trace semantics is defined by $\lg _{T}$ 's related by $\leq_{T}^{l}$ and the impossible futures trace semantics is defined by $\leq_{T}^{l}$.

\section{Deterministic Branching Observations}

Definition 12. 1. We say that a bgo is deterministic if for every node in it, its set of children $\left\{\left(a_{i}\right.\right.$, bgo $\left.\left._{i}\right)\right\}$ satisfies $a_{i} \neq a_{j}$ whenever $i \neq j$. We denote with $d B G O_{N}$ the set of deterministic observations in $B G O_{N}$.

2. The set of deteministic branching observations (dbgo for short) of a process $p$ is $d B G O_{N}(p)=B G O_{N}(p) \cap d B G O_{N}$.

3. We write $p \leq_{N}^{d b}$ q if $d B G O_{N}(p) \subseteq d B G O_{N}(q)$.

Like the linear observations, the set $d B G O_{N}(p)$ can be defined recursively and the corresponding semantics, compositionally.

Example 2. For the two processes $p=a(b c+b d)$ and $q=a b c+a b d$ we have that both deterministic observations $\langle\{a\},\{(a,\langle\{b\},\{(b,\langle\{c\}, \emptyset\rangle)\}\rangle)\}\rangle$ and $\langle\{a\},\{(a,\langle\{b\},\{(b$, $\langle\{d\}, \emptyset\rangle)\}\rangle)\}\rangle$ belong to $d B G O_{I}(p)$ and $d B G O_{I}(q)$. Indeed, that must be the case since it is easy to check that $d B G O_{I}(p)=d B G O_{I}(q)$.

In order to prove that dbgo's for the constraint $I$ characterize the possible world semantics we first recall the definition of that semantics in [6].

Definition 13. A deterministic process $p$ is a possible world of a process $q$ if $p \sqsubseteq_{R S} q$. The set of possible worlds of $p$ is denoted by $P W(p)$. We define the order $p \sqsubseteq P W q$ iff $P W(p) \subseteq P W(q)$.

When defining the possible worlds of a process we have to solve all the non-deterministic choices in it, each choice leading to one of its possible worlds. The same idea supports the selection of dbgo's to characterize this semantics: the non-deterministic branching observations in $B G O_{N}(p)$ are not present in $d B G O_{N}(p)$, where we have instead all the possible deterministic subtrees of every branching observation.

We call complete those observations that, for every node labeled by an offering $A$, have a branch labeled by each of the actions in $A$. 
Definition 14. The set of complete deterministic branching observations for the local observation function $L_{I}$ is the set cdBGO $\subseteq d B G O_{I}$ recursively defined as:

$-\langle\emptyset, \emptyset\rangle \in c d B G O_{I}$.

- $\left\langle A,\left\{\left(a\right.\right.\right.$, cdbgo $\left.\left.\left._{a}\right) \mid a \in A\right\}\right\rangle \in c d B G O_{I}$ for every $a \in A$ and $c d b g o_{a} \in c d B G O_{I}$.

For each $p \in B C C S P$ we define its set of complete deterministic branching observations $c d B G O_{I}(p)=d B G O_{I}(p) \cap c d B G O_{I}$

We also associate to a deterministic process $q$ its universal (complete deterministic) branching observation.

Definition 15. For a deterministic process $p$, its universal deterministic branching observation cdbgo $(p)$ is:

- $\operatorname{cdbgo}(\mathbf{0})=\langle\emptyset, \emptyset\rangle$.

- $\operatorname{cdbgo}\left(\sum_{a \in A} a p_{a}\right)=\left\langle A,\left\{\left(a, c d b g o\left(p_{a}\right)\right) \mid a \in A\right\}\right\rangle$.

The following result is then immediate.

Proposition 13. For every $p \in B C C S P, \operatorname{cdbgo}(p) \in c d B G O_{I}(p)$.

Lemma 1. For every $q \in P W(p)$, cdbgo $(q) \in c d B G O_{I}(p)$.

Lemma 2. For every process $q$ such that cdbgo $(q) \in c d B G O_{I}(p)$ we have $q \sqsubseteq_{R S} p$ and therefore $q \in P W(p)$.

Theorem 3. For all processes $p_{1}, p_{2} \in B C C S P, p_{1} \sqsubseteq_{P W} p_{2}$ iff $p_{1} \leq_{I}^{d b} p_{2}$.

Let us briefly consider the remaining new semantics definable by means of deterministic branching observations. It is clear that the corresponding orders verify $\leq_{N}^{b} \subseteq \leq_{N}^{d b} \subseteq \leq_{N}^{l}$, so that the associated semantics will be situated between the corresponding semantics defined by branching observations in $B G O_{N}$ and linear observations in $L G O_{N}$, as is the case for the possible worlds semantics, located between the ready simulation semantics and the ready trace semantics.

Admittedly, most of these semantics are rather strange and this is probably the reason why, as far as we know, they have not been previously considered. However, the simplest of them all, that corresponding to $N=U$, has properties similar to the possible worlds semantics and, in fact, can be defined by simply removing from its definition the " $R$ " in the condition $q \sqsubseteq R S p$. Hence, we can regard as possible worlds those deterministic implementations where we offer just a part of the action offered by the given process.

Definition 16. The partial possible worlds of a process $p$ are those deterministic processes that verify $q \sqsubseteq_{S} p$. We denote with $P W_{U}(p)$ the set of partial possible worlds of a process $p$ and define $p \sqsubseteq U P W q$ if $P W_{U}(p) \subseteq P W_{U}(q)$.

Proposition 14. For all processes $p_{1}, p_{2} \in$ BCCSP, $p_{1} \sqsubseteq U P W p_{2}$ iff $p_{1} \leq_{U}^{d b} p_{2}$.

Analogously, for any other constraint $N$ we could define the $N$-possible worlds using $\sqsubseteq_{N S}$, which in turn would be characterized using the observations in $d B G O_{N}$.

The extended spectrum, including also the diamond of linear semantics coarser than 2-nested simulation, can now be depicted as in Figures 3 and 4 




Fig. 3. The new linear time-branching time spectrum

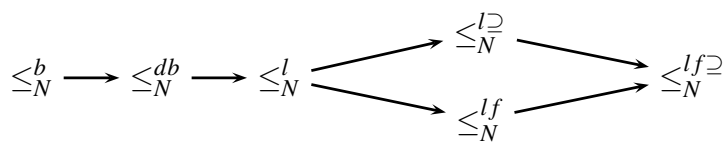

Fig. 4. Basic slice in the linear time-branching time spectrum

\section{Conclusion}

In this paper we have presented the first part of our unification work on the semantics for concurrency. We have seen that the branching-linear character of a semantics is the main fact to take into account in order to classify it properly. Indeed, this is not very surprising since the spectrum of semantics of concurrency was already called the linear time-branching time spectrum. The important result of our work is that all the branching semantics can be observationally characterized in a uniform way, so that the only difference between them is the local observation function $L_{N}$ used to watch the states of the processes. We have also uncovered the common structure of the diamonds under each simulation semantics, that corresponds to different orders on the sets of linear observations and are defined in the same way for all the constraints $N$. Finally, we found a single semantics in the spectrum defined by deterministic branching observations.

We think that this unification work sheds light on the structure of the spectrum. Besides, and more importantly, with the uniform descriptions of the semantics it will be much easier to prove general properties satisfied by all of them by means of parameterized proofs for generic constraints $N$, and also by considering the four orders defining the linear semantics corresponding to each constraint in a homogeneous way. In fact, as it was already mentioned in the introduction, we have found a common framework in which the axiomatizations of every semantics are particular cases of a couple of parametrized axioms; by using the uniform characterizations as definitions of the semantics we have already proved the soundness and completeness of the new axiomatizations for the semantics without having to resort to cumbersome proofs by cases. 


\section{References}

1. Boreale, M., Gadducci, F.: Processes as formal power series: A coinductive approach to denotational semantics. Theoretical Computer Science 360(1-3), 440-458 (2006)

2. de Frutos-Escrig, D., Gregorio-Rodríguez, C.: Universal coinductive characterizations of process semantics. In: 5th IFIP International Conference on Theoretical Computer Science. Springer Science and Business Media, vol. 273, pp. 397-412. Springer, Heidelberg (2008)

3. de Frutos-Escrig, D., Gregorio-Rodríguez, C., Palomino, M.: On the unification of process semantics: axiomatic semantics (submitted, 2008), http://maude.sip.ucm.es/ $\sim_{\text {miguept/ }}$

4. Hennessy, M.: Acceptance trees. Journal of the ACM 32(4), 896-928 (1985)

5. Jacobs, B.: Trace semantics for coalgebras. In: Adámek, J., Milius, S. (eds.) Proceedings of the Seventh Workshop on Coalgebraic Methods in Computer Science (CMCS 2004). Electronic Notes in Theoretical Computer Science, vol. 106. Elsevier, Amsterdam (2004)

6. van Glabbeek, R.J.: The linear time-branching time spectrum I: The semantics of concrete, sequential processes. In: Bergstra, J.A., Ponse, A., Smolka, S.A. (eds.) Handbook of process algebra, pp. 3-99. North-Holland, Amsterdam (2001)

7. Voorhoeve, M., Mauw, S.: Impossible futures and determinism. Information Processing Letters 80(1), 51-58 (2001) 